\title{
CARDIOGRAFÍA POR IMPEDANCIA EN LA EVALUACIÓN DEL PERFIL HEMODINÁMICO EN LA CARDIOPATÍA DILATADA. ESTUDIO TRANSVERSAL EN UN HOSPITAL PÚBLICO DE ECUADOR
}

\author{
IMPEDANCE CARDIOGRAPHY IN THE EVALUATION OF THE HEMODYNAMIC PROFILE IN \\ DILATED CARDIOPATHY. CROSS SECTIONAL STUDY IN A PUBLIC HOSPITAL IN ECUADOR
}

\author{
CARDIOGRAFIA POR IMPEDÂNCIA NA AVALIAÇÃO DO PERFIL HEMODINÂMICO NA DOENÇA \\ CARDÍACA EXPANDIDA. ESTUDO TRANSVERSAL NUM HOSPITAL PÚBLICO DO EQUADOR
}

\author{
MARÍA CAROLINA DUARTE MARTÍNEZ1', CARLOS ANDRÉS PEÑAHERRERA', ERNESTO PEÑAHERRERA PATIÑO² \\ 1 Universidad Católica de Santiago de Guayaquil, Guayaquil, Ecuador \\ 2 Hospital Luis Vernaza, Guayaquil, Ecuador
}

Resumen

Objetivos: describir el perfil hemodinámico de los pacientes del Ecuador con cardiopatía dilatada utilizando el sistema de cardiografía por impedancia, y comparar estos valores con los obtenidos de pacientes sanos. Metodología: 30 pacientes con diagnóstico previo de miocardiopatía dilatada en el Servicio de Cardiología del hospital Luis Vernaza fueron reclutados entre enero y junio de 2015. Fueron excluidos los pacientes con nefropatía crónica, cirrosis hepática o falla cardiaca grado IV de la New York Heart Association. Se midió las variables hemodinámicas mediante cardioimpedancia durante diez minutos. Posteriormente se realizó la misma medición a 30 controles voluntarios sin cardiopatía previa. Se calculó diferencia de medias mediante prueba T de Student y correlación de Pearson para evaluar las mediciones de función sistólica del ventrículo izquierdo. Resultados: la cardiopatía dilatada fue más común en varones (2:1), la principal causa fue la hipertensión crónica $(70 \%)$. Se obtuvieron diferencias estadísticamente significativas $(p<0,05)$ en las variables de volumen latido, índice latido, gasto cardiaco, índice cardiaco y resistencia periférica total; no existió diferencia estadísticamente significativa en las presiones arteriales, agua corporal total e impedancia basal. El índice Granov-Goor se correlacionó débilmente con la fracción de eyección ventricular. Conclusión: la hipertensión arterial crónica es la principal causa de cardiopatía dilatada en nuestro medio. Estos pacientes tienen disminuidos los patrones hemodinámicos de bomba cardiaca (disfunción sistólica), y aumentada la resistencia periférica total, como cabría esperarse. La cardioimpedancia es un método eficaz, cómodo y no invasivo en el abordaje de las patologías cardiovasculares. PALABRAS CLAVE: cardiografía de impedancia, cardiopatías, insuficiencia cardiaca, hemodinámica, estudios transversales, Ecuador.

Abstract

Objectives: to describe the hemodynamic profile of patients from Ecuador with dilated cardiomyopathy using the impedance cardiography system and to compare these values with those obtained from healthy patients. Methodology: 30 patients with previous diagnosis of dilated cardiomyopathy at Luis Vernaza hospital cardiology service were recruited between January and June 2015. Patients with chronic nephropathy, liver cirrhosis or Grade IV heart failure of the New York Heart Association were excluded. Hemodynamic variables were measured by cardioimpedance for 10 minutes. Subsequently, the same measurement was performed on 30 voluntary controls without previous heart disease. Mean difference was calculated by Student's t-test and Pearson's correlation to evaluate the measurements of left ventricular systolic function. Results: dilated heart disease was more common in males (2: 1), the main cause was chronic hypertension $(70 \%)$. Statistically significant differences $(\mathrm{p}<0.05)$ were found in the variables of beat volume, beat rate, cardiac output, cardiac index and total peripheral resistance; there was no statistically significant difference in arterial pressures, total body water, and baseline impedance. The Granov-Goor index was weakly correlated with the ventricular ejection fraction. Conclusion: chronic arterial hypertension is the main cause of dilated heart disease in our country. These patients have decreased hemodynamic heart pump patterns (systolic dysfunction), and increased total peripheral resistance, as would be expected. Cardioimpedance is an effective, comfortable and non-invasive method in the approach to cardiovascular diseases.

KEYWORDS: cardiography, impedance, heart diseases, heart failure, hemodynamics, cross-sectional studies, ecuador.

Resumo

Objetivos: descrever o perfil hemodinâmico dos pacientes com cardiomiopatia dilatada no Equador utilizando o sistema de cardiografia por impedância e comparar estes valores com os obtidos a partir de pacientes saudáveis. Métodología: 30 pacientes com diagnóstico prévio de cardiomiopatia dilatada no Servicio de Cardiologia do hospital Luis Vernaza foram recrutados entre janeiro e junho 2015. Foram excluídos pacientes com doença renal crônica, cirrose hepática ou insuficiência cardíaca grau IV da New York Heart Association. Variáveis hemodinâmicas foram medidos por cardioimpedancia durante 10 minutos. Mais tarde foi realizada a mesma medida a 30 voluntários sem doença cardíaca prévia. Foi calculada a diferença média pelas medições de teste te Student e de correlação de Pearson para avaliar a função sistólica do ventrículo esquerdo. Resultados: cardiomiopatia dilatada era mais comum no sexo masculino (2: 1), a principal causa foi a hipertensão crônica (70 \%). Se obtiveram diferenças estatisticamente significantes $(p<0,05)$ nas variáveis de volume batimento, a taxa de batimentos cardíacos, 0 vazao cardíaca, índice cardíaco e resistência periférica total; não houve diferença estatisticamente significativa na pressão arterial, água corporal total e impedância basal. 0 índice Granov-Goor foi fracamente correlacionado com fração de ejeção ventricular. Conclusão: a hipertensão arterial crônica é a principal causa de cardiomiopatia dilatada em nosso meio. Estes pacientes têm diminuído padrões hemodinâmicos de bomba cardíaca (disfunção sistólica) e aumentado a resistência periférica total, como seria de esperar. A cardioimpedancia é uma forma eficaz, conveniente e não invasiva no tratamento de método doenças cardiovasculares. PALABRAS-CHAVE: cardiografia de impedância, cardiopatias, insuficiência cardíaca, hemodinâmica, estudos transversais, equador. 


\section{INTRODUCCIÓN}

La enfermedad cardiovascular es la principal causa de mortalidad en Latinoamérica, es una de las regiones del mundo con la mayor cantidad de factores de riesgo. ${ }^{1}$ Se le adjudican casi el $50 \%$ de hospitalizaciones anuales, y el pronóstico suele ser variable debido a la implementación errónea o tardía del tratamiento. ${ }^{2}$ De acuerdo a estudios recientes, las poblaciones de Ecuador tienen una pobre salud cardiovascular, debido a la presencia de múltiples factores predisponentes y enfermedades asociadas. $^{3}$

Entre las enfermedades cardiovasculares de mayor morbimortalidad se encuentra la insuficiencia cardíaca congestiva (ICC). La causa más importante de ICC es la cardiopatía dilatada, principal motivo de trasplante cardíaco. ${ }^{4} \mathrm{El}$ entendimiento de los aspectos biomecánicos de la ICC es fundamental para evaluar el estado de la disfunción y por ende definir o reevaluar el tratamiento individualizado.

La medición correcta de los valores mecánicos del ventrículo izquierdo puede detectar de manera temprana alteraciones iniciales en cardiopatías, mejorando así el pronóstico de la enfermedad. 5,6 La cardiografía por impedancia mide la conductividad eléctrica del tórax y sus variaciones (impedancia), mediante un electrodo de entrada y uno de salida. ${ }^{7-9}$ Esta técnica permite la medición de variables funcionales como el gasto cardíaco, volumen sistólico, fluidos torácicos, función contráctil, entre otros; y al no ser invasiva ofrece una alternativa segura y confiable para la evaluación de enfermedades cardiovasculares. ${ }^{10}$ Existe relación entre los valores hemodinámicos reportados mediante cardioimpedancia, con los obtenidos mediante monitoreo invasivo. ${ }^{11}$

En Ecuador la cardiopatía dilatada es una causa frecuente de insuficiencia cardiaca y afecta negativamente la calidad de vida del paciente. ${ }^{12}$ La cardioimpedancia es una técnica accesible, de fácil implementación por los servicios de cardiología, que ofrece a los médicos seguridad en la evaluación hemodinámica, y a los pacientes un ambiente menos tenso y más confortable. ${ }^{13,14}$ No existen reportes estandarizados ni anecdóticos del uso de cardioimpedancia en el país, por lo que es imperativo realizar reportes de pacientes con diversas enfermedades cardiovasculares, para conocer la eficacia de esta herramienta diagnóstica en el medio local. Este estudio determinará si existe diferencia en los valores hemodinámicos de pacientes con cardiopatía dilatada en comparación a los no cardiópatas. Por otro lado, se podrá comprobar, la seguridad, facilidad de implementación y confiabilidad de la cardiografía por impedancia como técnica de abordaje no invasivo en la evaluación de enfermedades cardiovasculares.

\section{MATERIALES Y MÉTODOS}

Se realizó un estudio observacional de tipo transversal durante seis meses en el servicio de cardiología del hospital Luis Vernaza de la ciudad de Guayaquil, de enero a junio de 2015. El sistema de cardiografía por impedancia utilizado fue el Noninvasive Cardiac System $\left(\mathrm{NiCaS}^{\mathrm{TM}}\right)$ de NI Medical Ltd. ${ }^{15,16}$

Durante el tiempo del estudio se incluyó a 30 pacientes mayores de 18 años, de ambos géneros, con diagnóstico previo de cardiopatía dilatada y ecocardiografía realizada en el hospital. Se excluyó a los pacientes en estadio IV, según la escala de la New York Heart Association $(\mathrm{NYHA})^{17}$ para insuficiencia cardiaca, así como aquellos con diagnóstico de cirrosis hepática o enfermedad renal crónica en estadío 3 o mayor, enfermedades que causan una alteración no cardiogénica de los patrones hemodinámicos. Se obtuvo el peso, talla y presión arterial en reposo de los individuos seleccionados previo a la realización de la prueba y estos datos fueron ingresados en la pantalla del sistema $\mathrm{NiCa} \mathrm{S}^{\mathrm{TM}}$. Los electrodos de detección eléctrica se colocaron en muñeca izquierda y tobillo derecho, este último se cambió a muñeca derecha en los casos donde se detectaba señal baja, según el protocolo establecido. ${ }^{9,18,19}$ El sistema $\mathrm{NiCaS}^{\text {TM }}$ realiza mediciones hemodinámicas automáticamente cada 20 segundos; para la toma de cada paciente se realizó un registro de diez minutos para obtener un promedio adecuado de todas las mediciones obtenidas. De igual manera, se llevó a cabo la medición en 30 controles sin patología cardiovascular, pareados por edad y género.

La información obtenida fue recopilada inicialmente en una hoja de recolección de datos, utilizando las variables hemodinámicas reportadas por el sistema de cardioimpedancia, Tabla 1. Además, se incluyeron como variables: edad, género, causa de la cardiopatía dilatada (en caso de estar reportada), fracción de eyección media por ecocardiografía previa y péptido natriurético tipo B (BNP). Estas últimas tres variables no fueron consideradas para los controles sanos. 
El análisis estadístico se realizó con el software SPSS Statistics ${ }^{\circledR} 22$ (IBM ${ }^{\circledR}$ Corporation), se obtuvo la media, mediana, moda y desviación estándar (DS) de todas las variables hemodinámicas en ambos grupos de pacientes. Se calculó el porcentaje de cada etiología de cardiopatía dilatada en los pacientes incluidos para determinar su orden de frecuencia. El índice Granov-Goor (GCI) es una medida de cardioimpedancia que se relaciona con la función sistólica del ventrículo izquierdo, ${ }^{20,21}$ por lo que se comparó la correlación de este índice con la fracción de eyección mediante coeficiente de Pearson.

Posteriormente se realizó la prueba T de Student para determinar la diferencia de medias para cada variable entre el grupo de pacientes con cardiopatía y los controles sanos. Para estos cálculos se consideró una diferencia estadísticamente significativa a un valor $\mathrm{p}<0,05$. Todos los pacientes firmaron consentimiento informado de participación, dentro de los parámetros de la declaración de Helsinki.

\begin{tabular}{|c|c|c|c|}
\hline VARIABLE & $\begin{array}{r}\text { LÍMITE } \\
\text { INFERIOR } \\
\text { NORMAL* }\end{array}$ & $\begin{array}{r}\text { LÍMITE } \\
\text { SUPERIOR } \\
\text { NORMAL* }\end{array}$ & $\begin{array}{r}\text { UNIDAD DE } \\
\text { MEDIDA }\end{array}$ \\
\hline $\begin{array}{l}\text { Índice de masa corporal } \\
\text { (IMC) }\end{array}$ & 18 & 30 & $\mathrm{Kg} / \mathrm{m} 2$ \\
\hline Frecuencia cardiaca (FC) & 60 & 90 & $\begin{array}{l}\text { Latidos por } \\
\text { minuto (lpm) }\end{array}$ \\
\hline
\end{tabular}

\begin{tabular}{lccr}
\hline Frecuencia respiratoria (FR) & 8 & 24 & $\begin{array}{r}\text { Respiraciones por } \\
\text { minuto (RPM) }\end{array}$ \\
\hline $\begin{array}{l}\text { Presión arterial sistólica } \\
\text { (PAS) }\end{array}$ & 90 & 140 & $\mathrm{mmHg}$ \\
\hline $\begin{array}{l}\text { Presión arterial diastólica } \\
\text { (PAD) }\end{array}$ & 60 & 90 & $\mathrm{mmHg}$ \\
\hline $\begin{array}{l}\text { Presión arterial media } \\
\text { (PAM) }\end{array}$ & 70 & 105 & $\mathrm{mmHg}$ \\
\hline
\end{tabular}

\begin{tabular}{lccr}
\hline Volumen latido (VL) & 60 & 130 & $\mathrm{ml}$ \\
\hline Índice latido (IL) & 35 & 65 & $\mathrm{ml} / \mathrm{m} 2$
\end{tabular}

\begin{tabular}{lccr}
\hline Gasto cardiaco (GC) & 4 & 8 & $\mathrm{~L} / \mathrm{min}$ \\
\hline Índice cardiaco (IC) & 2,5 & 4 & $\mathrm{~L} / \mathrm{min} / \mathrm{m} 2$
\end{tabular}

\begin{tabular}{lccrr}
\hline Índice granov-goor (GGI) & 10 & - & $\begin{array}{r}\text { Índice de función } \\
\text { del VI }\end{array}$ \\
\hline Resistencia periférica total & 770 & 1500 & dinas*s/cm5 $^{*}$
\end{tabular}
(RPT)

\begin{tabular}{lrrr}
\hline Impedancia basal (R) & 250 & 750 & Ohmios (ohm) \\
\hline Porcentaje de agua corporal & 34,5 & 46,5 & Porcentaje (\%)
\end{tabular}
total (\% act)

*Según el manual de operaciones del sistema NICASTM. ${ }^{22}$ El equipo está configurado según estos parámetros.
RESULTADOS

En el hospital Luis Vernaza, de enero a junio de 2015, se evaluaron 56 pacientes con cardiopatía dilatada, de los que no se incluyó a 15 , por no tener ecocardiografía realizada. Un total de 11 pacientes de este grupo fueron excluidos tras aplicar criterios de exclusión (cinco se encontraban en grado IV de la NYHA y seis padecían de enfermedad renal crónica severa, de los cuales tres tenían también cirrosis hepática) dejando 30 individuos en el grupo de estudio. Posteriormente se reclutó a 30 controles sanos voluntarios. En el grupo de pacientes con cardiopatía dilatada, el $67 \%$ de los pacientes fueron de género masculino y el $33 \%$ corresponden al género femenino. La media de edad fue 62,1 (DS \pm 14 ), la mediana 64,5 y la moda 65 . Por otro lado, en el grupo de controles sanos hubo un $57 \%$ de sujetos masculinos y $43 \%$ de femeninos, mientras que la media de edad fue de 56,23 (DS \pm 14 ) y la mediana 55 , al igual que la moda.

Los pacientes con cardiopatía dilatada tuvieron en el $43 \%$ un IMC dentro de parámetros normales, entre 18 y $25 \mathrm{Kg} / \mathrm{m} 2$, mientras que la frecuencia cardiaca, frecuecia respiratoria y presión arterial se encontraron en valores normales entre el 70 y $80 \%$ de pacientes. La etiología más frecuente de cardiopatía dilatada fue la hipertensión arterial crónica (70 \%), los porcentajes de cada causa reportada se presentan en la (Figura 1). El estadio más frecuente en la escala NYHA fue el $3(40 \%)$, correspondiente a fatiga y disnea al realizar esfuerzos leves. A 25 pacientes se les midió BNP, variable que tuvo un promedio de 9941,6 ( \pm 9232).

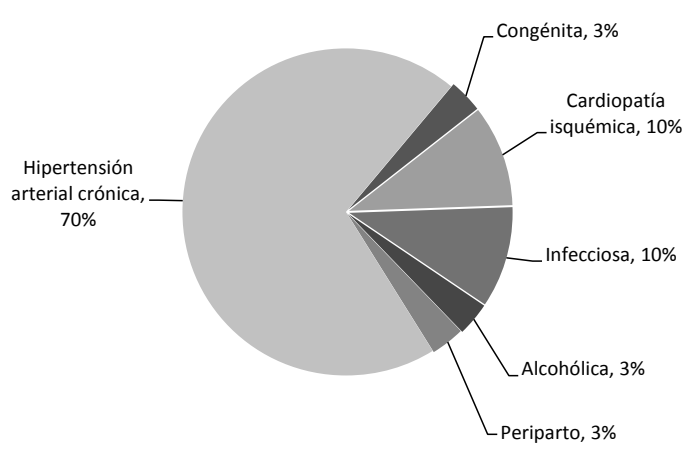

Figura 1. Etiología de la cardiopatía dilatada según porcentaje.

Se realizó la prueba $\mathrm{T}$ de Student a los datos obtenidos de ambos grupos de sujetos para determinar la diferencia de medias y la significancia estadística de cada una de las variables hemodinámicas obtenidas mediante 
cardiografía por impedancia. Estos resultados se presentan en la tabla 2 , donde se muestra la diferencia de medias en cada variable junto a su valor $\mathrm{p}$.

\begin{tabular}{|c|c|c|c|c|}
\hline VARIABLE & $\begin{array}{r}\text { MEDIA (DS) } \\
\text { GRUPO } \\
\text { CASOS }\end{array}$ & $\begin{array}{r}\text { MEDIA (DS) } \\
\text { GRUPO } \\
\text { CONTROLES }\end{array}$ & $\begin{array}{r}\text { DIFEREN- } \\
\text { CIA DE } \\
\text { MEDIAS* }\end{array}$ & $\begin{array}{r}\text { VALOR } \\
\mathbf{P}\end{array}$ \\
\hline $\begin{array}{l}\text { Presión arterial sistólica } \\
(\mathrm{mmHg})\end{array}$ & $119(23,8)$ & $124,7(12,5)$ & $-5,63$ & 0,26 \\
\hline $\begin{array}{l}\text { Presión arterial diastólica } \\
(\mathrm{mmHg})\end{array}$ & $72,8(14,3)$ & $72,4(9,7)$ & 0,43 & 0,89 \\
\hline $\begin{array}{l}\text { Presión arterial media } \\
(\mathrm{mmHg})\end{array}$ & $87,9(15)$ & $89,5(8,7)$ & $-1,53$ & 0,63 \\
\hline Volumen latido $(\mathrm{ml})$ & $55,1(13,2)$ & $83,7(14,9)$ & $-28,6$ & $<0,01$ \\
\hline Índice latido (ml/m²) & $32,7(7,2)$ & $48(9,1)$ & $-15,23$ & $<0,01$ \\
\hline Gasto cardiaco (L/min) & $4,3(1)$ & $5,9(1,1)$ & $-1,62$ & $<0,01$ \\
\hline Índice cardiaco $\left(\mathrm{L} / \mathrm{min} / \mathrm{m}^{2}\right)$ & $2,6(0,6)$ & $3,4(0,7)$ & $-0,84$ & $<0,01$ \\
\hline $\begin{array}{l}\text { Resistencia periférica total } \\
\left(\text { dinas }^{*} \mathrm{~s} / \mathrm{cm}^{5}\right)\end{array}$ & $\begin{array}{r}2187,4 \\
(2114,3) \\
\end{array}$ & $1269,7(288)$ & 917,6 & 0,02 \\
\hline $\begin{array}{l}\text { Índice Granov-Goor (índice } \\
\text { de función del VI) }\end{array}$ & $9,5(2,7)$ & $14,6(2,9)$ & -5 & $<0,01$ \\
\hline Agua corporal total (\%) & $54,3(12,2)$ & $49,2(10,9)$ & 5,05 & 0,09 \\
\hline Impedancia basal (ohm) & $366,368,2)$ & $386,9(71,4)$ & $-20,63$ & 0,26 \\
\hline
\end{tabular}

* La diferencia de medias es el resultado de la media del grupo de casos tras restarle la media del grupo control.

El índice Granov-Goor (GGI) de los pacientes con cardiopatía dilatada fue pareado con la fracción de eyección obtenida del reporte de ecocardiografía de los 30 casos, para observar el comportamiento de esta variable en la evaluación de la función sistólica del ventrículo izquierdo. La correlación de Pearson de ambas variables dio un resultado positivo de $\mathrm{r}$ : 0,33 (valor $\mathrm{p}=0,038$ ). Estos resultados aparecen graficados en la figura 2 , donde se observa que la mayoría de los pacientes dilatados se encuentran en el cuadrante por debajo del límite inferior de ambas variables.

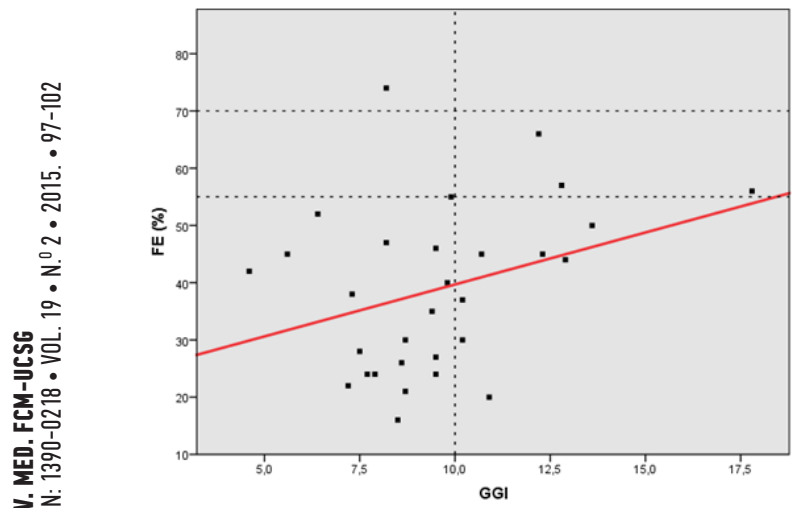

Figura 2. Gráfico de dispersión de la correlación entre fracción de eyección ventricular e índice de Granov-Goor.
DISCUSIÓN

En Ecuador, la ICC es una causa frecuente de hospitalizaciones debido a la alta prevalencia de enfermedades que la causan, y posee la segunda tasa más alta de América Latina. ${ }^{22,23}$ La forma más frecuente de presentación es la cardiopatía dilatada crónica, la cual tiene entre sus principales causas a la hipertensión arterial, la cardiopatía isquémica, las malformaciones congénitas, y ciertas causas infecciosas que son endémicas a la región, entre otras. ${ }^{1,2,24}$

La mortalidad de la insuficiencia cardiaca alcanza el 6,3\% de todas las causas de muerte en Latinoamérica. ${ }^{2}$ Su diagnóstico temprano resulta significativo en el desarrollo de la misma, y por ende tiene impacto en la tasa de fallecimiento por año. La facilidad de acceso a diferentes técnicas diagnósticas, asegura el seguimiento de pacientes con cardiopatía dilatada y el inicio de un tratamiento oportuno y adecuado. ${ }^{24}$

Hasta ahora en Ecuador sólo se ha podido obtener las mediciones hemodinámicas de los pacientes con insuficiencia cardiaca mediante técnicas invasivas como el cateterismo cardíaco. ${ }^{7}$ La aparición de técnicas no invasivas diagnósticas como la cardiografía por impedancia facilita el abordaje de estos pacientes en un marco de tiempo más corto, con menor incomodidad para el paciente, y el mejor control de las exacerbaciones.,11,13,15 El análisis de un grupo de pacientes con cardiopatía dilatada mediante cardioimpedancia en el presente estudio nos permite observar, entre otras cosas, la implementación y beneficios de esta nueva herramienta diagnóstica en nuestro medio.

En el presente estudio se observó que la cardiopatía dilatada fuemás frecuenteen pacientes masculinos con una relación 2:1, y se presentó principalmente alrededor de los 60 años, lo que coincide con los datos descritos en reportes previos. ${ }^{3,4,25}$ De las diversas causas de cardiopatía dilatada se encontró que la más prevalente fue la hipertensión arterial, y en menor proporción la cardiopatía isquémica, las causas infecciosas, congénita, alcohólica y la miocardiopatía, mientras que no se observó ningún caso provocado por otros factores reconocidos como los metabólicos o farmacológicos. ${ }^{4,26}$

En estudios previos realizados en el servicio de cardiología, ya se ha mostrado a la hipertensión arterial como la principal causante de otras enfermedades cardiovasculares como arritmias, y la cardiopatía dilatada no es la excepción ${ }^{27}$. Los valores de BNP en los pacientes a quienes se les midió este parámetro tuvieron variaciones muy amplias, y 
aunque generalmente estuvieron por encima del límite superior normal, no se relacionaron con la severidad de la enfermedad. La mayoría de pacientes evaluados se encontraban en grado III de NYHA; sin embargo, hay que tener en cuenta que no se incluyeron pacientes con grado NYHA IV.

Encuantoalosparámetros hemodinámicosobtenidos mediante cardioimpedancia, seobservó diferencias significativas con respecto a los controles sanos en las variables de volumen, latidoy gasto cardiaco, que se relacionan con la función de bomba del corazón; índice latido e índice cardíaco, que se refieren a la distribución del bombeo en relación a la superficie corporal, y en los valores de resistencia periférica total. La dilatación progresiva y excéntrica de las cavidades cardíacas, altera la capacidad contráctil de los ventrículos, lo que disminuye la función sistólica del corazón al no tener fuerza suficiente para bombear una cantidad adecuada de sangre hacia la aorta. ${ }^{5,15,16}$ Esta alteración se corrobora con los resultados obtenidos en este estudio, donde tanto el volumen sanguíneo expulsado en cada latido, como la cantidad de sangre bombeada por minuto, así como sus variantes indexadas, tuvieron un valor menor comparado con pacientes sin cardiopatía dilatada.

La resistencia periférica total resultó aumentada en el grupo de estudio, lo que apoya el hecho de que la hipertensión arterial es la causa más frecuentedecardiopatía dilatada, pues la resistencia periférica elevada tiene un rol en la fisiopatología de la hipertensión. ${ }^{28,29}$ No se encontró diferencia estadísticamente significativa en presión arterial (sistólica, diastólica y media), porcentaje de agua corporal total, e impedancia basal.

La falta de diferencia en presiones arteriales entre los dos grupos, a pesar de la alta prevalencia de hipertensión, puede ser debida al tratamiento diurético que reciben los pacientes con cardiopatía dilatada, loquedisminuyeel volumenintravascular; aunque la resistencia periférica se vea aumentada en el grupo de casos. Este factor también puede alterar el porcentaje de agua corporal total y los valores de impedancia basal de las arterias., 4,29,30

Finalmente, se encontró una correlación positiva, débil pero significativa entre el GGI y la fracción de eyección; este resultado conlleva a suponer la utilidad de la cardioimpedancia como método de apoyo en cardiopatías en las que resulte relevante conocer la función del ventrículo izquierdo, pero no reemplazaa los datos obtenidos porecocardiografía. ${ }^{7,21}$
Entre las posibles limitaciones del estudio se registra el hecho de que no se obtuvieron valores hemodinámicos mediante cateterismo cardiaco para realizar una comparación con los obtenidos mediantecardioimpedancia, ya que sóloun pequeño número de los pacientes estudiados habían sido sometidos a esta intervención. .,30

Un futuro estudio que compare ambas técnicas, no invasiva frente a invasiva, será útil para determinar con mayor validez las mediciones impedanciocardiográficas. La muestra depacientes con cardiopatía dilatada es pequeña ya que se buscó principalmente describir el patrón hemodinámico de los individuos con la enfermedad, por lo que las próximas investigaciones deberán enfocarse en trabajar con grupos mayores de pacientes.

Los resultados obtenidos parecen concordar con lo descrito para la fisiopatología de la enfermedad, lo que indica que la cardioimpedancia da mediciones eficaces sobre la biomecánica cardiaca y sugiere una buena generabilidad para aplicarla a otras enfermedades cardiovasculares.

CONCLUSIONES

Serealizóuna descripción del patrón hemodinámico en pacientes con cardiopatía dilatada medido por cardioimpedancia, este es el primer informe que se desarrolla en Ecuador usando este apoyo diagnóstico, y es el primero en Latinoamérica que utiliza el sistema $\mathrm{NiCaS}^{\mathrm{TM}}$.

Los patrones hemodinámicos que se mostraron alterados en la medición por cardioimpedancia, demuestran la afectación de bombeo y resistencia periférica, descritos en la literatura médica para pacientes con cardiopatía dilatada; lo que corrobora la veracidad de los valores obtenidos.

La cardiografía por impedancia demostró ser, a lo largo del estudio, un método benévolo y atractivo para los pacientes, al ser no invasivo tuvo un mejor recibimiento por parte de los individuos y sus familiares. Por su comodidad y velocidad en el reporte de los resultados se muestra como una técnica muy útil en el abordaje de estos pacientes por lo que se sugiere su implementación en los servicios de cardiología.

CONFLICTOS DE INTERESES

Los autores declaran no tener ningún conflicto de interés, concerniente a la realización de este estudio y los materiales utilizados. No se ha recibido financiamiento externo para la realización de este estudio. 


\section{REFERENCIAS BIBLIOGRÁFICAS}

1. Hernández-Leiva E. Epidemiología del síndrome coronario agudo y la insuficiencia cardiaca en Latinoamérica. Rev Esp Cardiol. 2011 Jul;64, Supplement 2:34-43.

2. Bocchi EA. Heart failure in South America. Curr Cardiol Rev. 2013 May;9(2):147-56.

3. Del Brutto OH, Santamaría M, Ochoa E, Peñaherrera E, Santibáñez R, Pow-Chon-Long F, et al. Population-based study of cardiovascular health in Atahualpa, a rural village of coastal Ecuador. Int J Cardiol. 2013 Sep 30;168(2):1618-20.

4. LakdawalaNK, WinterfieldJR, FunkeBH. Dilatedcardiomyopathy. Circ Arrhythm Electrophysiol. 2013 Feb;6(1):228-37.

5. Bustamante J, Valbuena J. Biomecánica de la falla cardíaca. Insufic Cardíaca. 2008 Dec;3(4):173-83.

6. ModestoK, SenguptaPP.Myocardialmechanicsincardiomyopathies. Prog Cardiovasc Dis. 2014 Aug;57(1):111-24.

7. AlmeidaJuniorCL, XavierSS, GarciaMI, ClausellN. Hemodynamic assessment in heart failure: role of physical examination and noninvasivemethods. Arq Bras Cardiol. 2012 Jan;98(1):e15-21.

8. CybulskiG. Ambulatory Impedance Cardiography-TheSystems and their Applications. Berlin Heidelberg: Springer-Verlag; 2011.

9. WoltjerHH, Bogaard HJ, deVries PM. Thetechniqueofimpedance cardiography. Eur Heart J. 1997 Sep;18(9):1396-403.

10. RosenbergP, YancyCW. Noninvasiveassessmentofhemodynamics: an emphasis on bioimpedancecardiography. CurrOpin Cardiol. 2000 May;15(3):151-5.

11. Kamath SA, Drazner MH, Tasissa G, Rogers JG, Stevenson LW, Yancy CW. Correlation of impedance cardiography withinvasive hemodynamic measurements in patients with advanced heart failure: the BioImpedance CardioGraphy (BIC) substudy of the Evaluation Study of Congestive Heart Failure and Pulmonary Artery Catheterization Effectiveness (ESCAPE) Trial. Am Heart J. 2009 Aug;158(2):217-23.

12. Muñoz Yépez SL, Gabela J. Caso clínico de educación médica paciente masculino adulto mayor diabético con insuficiencia cardiaca y miocardiopatía dilatada [Internet]. [Quito, Ecuador]: Universidad San Francisco de Quito; 2014 [cited 2015 Jul 28]. Availablefrom: http://repositorio.usfq.edu.ec/handle/23000/3513.

13. AlbertNM. Bioimpedancetopreventheartfailurehospitalization. Curr Heart Fail Rep. 2006 Sep;3(3):136-42.

14. Gilbert J, Lazio L. Managing congestive heart failure with thoracic electrical bioimpedance. AACN Clin Issues. 1999 Aug;10(3):400-5.

16. CotterG, Torre-AmiotG, VeredZ, KaluskiE. Whole-bodyelectrical bio-impendance is accurate in non invasive determination of cardiac output: a thermodilution controlled, prospective, double blind evaluation. Eur J Heart Fail Suppl. 2004 Jun 1;3:19-19.

17. Goldman L, Hashimoto B, Cook EF, Loscalzo A. Comparative reproducibilityand validity of systems forassessing cardiovascular functional class: advantages of a new specific activity scale. Circulation. 1981 Dec 1;64(6):1227-34.

18. NI Medical Ltd. User manual for new NI Medical LTD's model NICaS CS - With Software version 3.58. NI Medical Ltd., Ireland; 2011.

19. Sherwood A, Allen MT, Fahrenberg J, Kelsey RM, Lovallo WR, van Doornen LJ. Methodological guidelines for impedance cardiography. Psychophysiology. 1990 Jan;27(1):1-23.

20. Granov E, Granov I, Goor D, Frinerman E. Method and system for use in monitoring left ventricular dysfunction [Internet]. US8306611 B2, 2012 [cited 2015 Jul 4]. Available from: http:// www.google.com/patents/US8306611.

21. Rozenman Y, Rotzak R, Patterson RP. Detection of left ventricular systolic dysfunction using a newly developed, laptop based, impedance cardiographic index. Int J Cardiol. 2011 Jun 2;149(2):248-50.

22. Instituto Nacional de Estadística y Censos. Estadísticas de Camas y Egresos Hospitalarios 2014 [Internet]. 2014 [cited 2015 Aug 6]. Available from: http://www.ecuadorencifras.gob.ec/ camas-y-egresos-hospitalarios/.

23. Grupo de Estudios de Insuficiencia Cardíaca (GEIC) del DepartamentodeClínica dela Sociedad Brasilera de Cardiología y el Departamento de Directivas de la Sociedad Brasilera de Cardiología. Consenso: Ia Directiva Latinoamericana para la evaluación y conducta en la insuficiencia cardíaca descompensada: Parte I. Insufic Cardíaca. 2006 Mar;1(1):2-10.

24. Bocchi EA, Arias A, Verdejo H, Diez M, Gómez E, Castro P, et al. The reality of heart failure in Latin America. J Am Coll Cardiol. 2013 Sep 10;62(11):949-58.

25. Fairweather D, Cooper LT, Blauwet LA. Sex and gender differences in myocarditis and dilated cardiomyopathy. Curr Probl Cardiol. 2013 Jan;38(1):7-46.

26. Kawai C, Matsumori A. Dilated cardiomyopathy update: infectious-immune theory revisited. Heart Fail Rev. 2013 Nov;18(6):703-14.

27. Peñaherrera-OviedoC, TettamantiD. Causalidad defibrilación auricularenpacientesadultosatendidosenelhospitalLuisVernaza de Guayaquil, período 2011-2013. Rev Med. 2014;18(3):153-9.

28. Summers RL, Shoemaker WC, Peacock WF, Ander DS, Coleman TC. Bench to Bedside: Electrophysiologic and Clinical Principles of Noninvasive Hemodynamic Monitoring Using Impedance Cardiography. Acad Emerg Med. 2003 Jun 1;10(6):669-80.

29. Albert NM. Bioimpedance cardiography measurements of cardiac output and other cardiovascular parameters. Crit Care Nurs Clin North Am. 2006 Jun;18(2): 195-202, x.

30. Acquatella H. Miocardiopatía dilatada: avances recientes y tratamientoactual. RevEspCardiol. 2000 Apr 2;53(Supl. 1):19-27. 